\title{
Teor e composição química de óleo essencial de cidró em função da sazonalidade e horário de colheita
}

\author{
Dalva Paulus; Raquel Valmorbida; Ezequiel Toffoli; Gilmar Antônio Nava \\ ${ }^{1}$ UTFPR, C. Postal 157, 85660-000 Dois Vizinhos-PR; dalvaufsmdeutch@yahoo.com.br (autor para correspondência); \\ raquelvalmorbida@yahoo.com.br; toffoliezequiel@yahoo.com.br; gilmarnava@utfpr.edu.br
}

\begin{abstract}
RESUMO
O cidró (Aloysia triphylla) é uma planta medicinal de porte arbustivo, com folhas aromáticas que possuem óleo essencial rico em citral, e essa substância confere grande importância à indústria farmacêutica e cosmética. Objetivou-se avaliar o teor e os componentes do óleo essencial do cidró em função da sazonalidade e horário de colheita. O experimento foi conduzido na área experimental da Universidade Tecnológica Federal do Paraná, Campus Dois Vizinhos, de março de 2010 a setembro de 2011. O delineamento experimental utilizado foi de blocos ao acaso em esquema fatorial onde os fatores foram realizados nas épocas de extração (outubro/2010 a setembro/2011) e horários de colheita (8:00, 11:00, 14:00, 16:00 e 18:00 horas). Foram analisados o teor e composição química do óleo essencial, extraído pela técnica de hidrodestilação, utilizando o aparelho de Clevenger e analisado em cromatografia gasosa e espectofotometria de massa (CG/ EM). O mês de fevereiro resultou em maior teor de óleo essencial de cidró nos horários de colheita das 16:00 e 18:00 horas, provavelmente ocasionado pelas condições climáticas favoráveis e a presença de folhas jovens geneticamente ativas. Os constituintes majoritários do óleo essencial foram geranial (36,96\%), neral $(29,31 \%)$, nerolidol $(24,34 \%)$ e limoneno $(15,77 \%)$.
\end{abstract}

Palavras-chave: Aloysia triphylla, variação sazonal, citral.

\begin{abstract}
Content and chemical composition of essential oil of cidró depending on seasonality and harvest time

The cidró (Aloysia triphylla) is a medicinal plant shrub with aromatic leaves, which have essential oil rich in citral; this substance has great importance in pharmaceutical and cosmetic industries. The objective of the study was to evaluate the content and components of the essential oil of cidró depending on seasonality and harvest time. The experiment was carried out in the experimental area of the Universidade Tecnológica Federal do Paraná, Campus Dois Vizinhos, from March 2010 to September 2011. The experimental design was randomized blocks in factorial where the factors consisted of times of extraction (October 2010 to September 2011) and harvest times (8:00, 11:00, 14:00, 16:00 and 18:00 o'clock). We evaluated content and chemical composition of essential oil. The essential oil was extracted by hydrodistillation technique, using apparatus Clevenger and analyzed by gas chromatography and mass spectrophotometry (GC/MS). The month of February resulted in higher levels of essential oil of cidró at the harvest times of 16:00 and 18:00 o'clock, probably due to favorable climatic conditions and the presence of genetically active young leaves. The major constituents of the essential oil were geranial (36.96\%), neral (29.31\%), nerolidol (24.34\%) and limonene $(15.77 \%)$.
\end{abstract}

Keywords: Aloysia triphylla, seasonal variation, citral.

\section{(Recebido para publicação em 15 de maio de 2012; aceito em 5 de abril de 2013)} (Received on May 15, 2012; accepted on April 5, 2013)

\begin{abstract}
$\mathrm{A}^{4}$ Aloysia triphylla (cidró) é um arbusto grande (2 a 3 metros), muito ramificado e ereto. É uma planta adstringente e aromática, provavelmente originária do Chile. É rico em óleo volátil, agindo como sedativo brando, e também ajuda na digestão e contra resfriados. Em aromaterapia é usada contra problemas nervosos e digestivos e para acnes. Na culinária é servida como recheio de bolos, no preparo de licores, sucos, pães e para dar aroma às carnes (Lorenzi \& Matos, 2008).

Suas folhas retêm muito bem seu aroma de citral, mesmo depois da secagem, tornando-se um componente indispensável nos potpourris muito
\end{abstract}

empregados para aromatizar ambientes. Possui também propriedades inseticidas e bactericidas (Lorenzi \& Matos, 2008). Além do seu uso como perfume, o citral é empregado na síntese de ionona (perfume da violeta), beta-caroteno e vitamina A (Czepak \& Cruciol, 2003).

O Brasil tem lugar de destaque na produção de óleos essenciais, ao lado da Índia, China e Indonésia, que são considerados os quatro grandes produtores mundiais. O Paraná é responsável por 90\% da produção nacional de plantas medicinais e aromáticas com aproximadamente dois mil hectares plantados, correspondendo a mais de $70 \%$ das espécies botânicas utilizadas pela indústria farmacêutica, de cosméticos, corantes, chás e de bebidas (Negrelle et al., 2005).

A biossíntese dos óleos essenciais é influenciada por fatores climáticos como fotoperíodo, temperatura, umidade, precipitação e intensidade de radiação solar, que podem determinar a época ideal de colheita ou o local de cultivo, onde poderá se obter maior quantidade de óleo essencial e do princípio ativo desejado. O estádio de desenvolvimento da planta também interfere na produção de metabólitos secundários (Taveira et al., 2003).

A influência da sazonalidade e horário de colheita sobre a produção de óleo essencial do cidró foi verificada 
por Brant et al. (2008) em Lavras-MG, onde os autores constataram maior teor de óleo essencial $(0,271 \%)$ no mês de abril e os melhores horários de colheita foram 8 e 16 horas.

Identificar o melhor horário de coleta das plantas medicinais e de maior produção de óleo essencial torna-se um aspecto relevante na produção de óleos essenciais, pelo fato de ocorrerem, durante o dia, oscilações nos componentes climáticos, principalmente temperatura e luminosidade (Brant et al., 2009). Diante da escassez de informações na literatura sobre o cidró, justifica-se a realização de estudos sobre a influência da sazonalidade e horários de colheita ao longo do dia, sendo que essas informações são de grande importância para o produtor de óleo essencial no momento da colheita. O objetivo foi avaliar o teor e os componentes químicos do óleo essencial do cidró em função da sazonalidade e horário de colheita.

\section{MATERIAL E MÉTODOS}

O trabalho foi desenvolvido na área experimental da Universidade Tecnológica Federal do Paraná, Campus Dois Vizinhos, de março de 2010 a setembro de 2011. O clima da região é classificado como Cfa subtropical úmido, sem estação seca definida e temperatura média do mês mais quente de $22^{\circ} \mathrm{C}$. $\mathrm{O}$ solo local é do tipo Nitossolo Vermelho Distroférrico (Bhering et al., 2008). A análise do solo apresentou como características químicas na camada de 10 a $20 \mathrm{~cm}$ de profundidade: $\mathrm{pH}$ em água: 5,70; M.O. $\left(\mathrm{g} \mathrm{dm}^{-3}\right)$ 41,55; P, Cu, Fe, Zn, $\mathrm{Mn}\left(\mathrm{mg} \mathrm{dm}^{-3}\right)$ : 31,29; 6,67; 19,67; 7,89; 197,26, respectivamente; $\mathrm{Ca}, \mathrm{Mg}, \mathrm{K}$, $\mathrm{SB}, \mathrm{H}+\mathrm{Al}\left(\mathrm{cmol} \mathrm{dm}^{-3}\right): 8,47 ; 2,78 ; 1,10$; 12,$35 ; 3,97$, respectivamente; $\mathrm{V}(\%)=$ 75,67 . Os tratos culturais utilizados na manutenção das plantas foram capina manual e irrigação por microaspersão.

As mudas de cidró foram produzidas em casa-de-vegetação situada no setor de olericultura da UTFPR. As estacas apicais de cinco $\mathrm{cm}$ de comprimento e dois $\mathrm{mm}$ de diâmetro foram obtidas a partir de planta matriz e enraizadas em bandejas de poliestireno expandido de 72 células, contendo substrato comercial.
As plantas com dez centímetros de altura foram transplantadas em 28 de março de 2010, em covas de 30x30 cm, com espaçamento $1 \times 1 \mathrm{~m}$ entre linhas e entre plantas.

O delineamento experimental utilizado foi de blocos ao acaso, sendo estudados os efeitos de 12 épocas de extração (outubro/2010 a setembro de 2011) que corresponderam a 213 , 247, 270, 300, 334, 368, 398, 430, 460, 521,550 e 581 dias após o transplantio (DAT), respectivamente e cinco horários de colheita: 8:00, 11:00, 14:00, 16:00 e 18:00 horas, em esquema fatorial de $12 \times 5$, com 10 repetições. Não foram realizadas extrações de óleo essencial no mês de julho, pois as plantas estavam sem folhas. Cada parcela experimental foi composta de 20 plantas de cidró, sendo consideradas cinco plantas úteis.

O teor de óleo essencial foi obtido de material fresco (folhas) por meio de extração dos componentes voláteis realizado em aparelho modificado do tipo Clevenger (método de hidrodestilação). Foram utilizadas amostras compostas (um galho por planta, nas regiões apical, mediana e basal) de 70 $\mathrm{g}$ de folhas frescas sadias, picadas em frações de aproximadamente $1 \mathrm{~cm}$. O tempo de extração foi de duas horas. Após a obtenção do óleo essencial puro foram medidas a massa e o teor calculado pela fórmula: $\mathrm{T} \%=$ massa do óleo (g) / 70g x 100.

Determinou-se a constituição química do óleo essencial, com três repetições dos meses de outubro, novembro, dezembro de 2010; janeiro, fevereiro, março, abril, maio, junho, agosto e setembro de 2011, no horário de extração das 8:00 horas da manhã em Cromatógrafo a Gás HP5890 equipado com detector por ionização de chamas. Utilizou-se uma coluna BP-1 (SGE) $25 \mathrm{~m} \mathrm{x} \mathrm{0,25} \mathrm{mm}$ com gradiente de temperatura $60^{\circ} \mathrm{C}, 1$ min, $3^{\circ} \mathrm{C} / \mathrm{min}$ até $220^{\circ} \mathrm{C}$; injetor (split de $1 / 50$ ) a $220^{\circ} \mathrm{C}$ e detector a $220^{\circ} \mathrm{C}$. Hidrogênio como gás de arraste $\left(2 \mathrm{~mL} \mathrm{~min}^{-1}\right)$ e volume de injeção de $1 \mu \mathrm{L}$. Amostras foram diluídas a 1,0\% em clorofórmio. A identificação dos picos foi feita por cálculo de índice de retenção com padrões de hidrocarbonetos lineares de C10 a C18 e comparação com dados de literatura (Adams, 2001).
Os dados meteorológicos (precipitação, temperatura, umidade relativa do ar e radiação solar) foram obtidos da estação meteorológica localizada próxima à unidade experimental.

Os resultados do teor e da composição química do óleo essencial foram submetidos à análise de variância com auxílio do programa "SAS" (SAS Institute, 1999). As variâncias dos tratamentos foram testadas quanto à homogeneidade pelo teste de Bartlett e as médias comparadas pelo teste de Tukey a $5 \%$ de probabilidade.

\section{RESULTADOS E DISCUSSÃO}

Ocorreu interação significativa entre horário e mês de colheita. No horário de colheita das 8:00 horas, os meses de janeiro, fevereiro e novembro apresentaram os maiores valores de teor de óleo essencial; os menores teores de óleo essencial foram obtidos nos meses de maio e junho, os demais meses não diferiram estatisticamente para esse horário de colheita.

As diferenças do teor de óleo essencial entre os meses de colheita sugerem ser decorrentes da influência dos fatores ambientais de temperatura e radiação. Verificou-se que no horário das 8:00 horas nos meses de janeiro, fevereiro e novembro, as temperaturas médias foram de 23,$6 ; 23,3$ e $21,0^{\circ} \mathrm{C}$; precipitação de 198,8; 186 e 272,6 mm; radiação de 995,$1 ; 830,1$ e $1070,5 \mathrm{~kJ} \mathrm{~m}^{-2}$ (Tabela 2). Verificou-se que a precipitação nesse período foi adequada, sem excesso ou escassez de chuvas, favorecendo o crescimento das plantas. $\mathrm{O}$ estresse hídrico pode aumentar, diminuir ou não alterar a produção de metabólitos secundários (Carvalho \& Casali, 1999). No presente estudo, a precipitação associada às condições de temperatura e radiação favoreceram a síntese do metabolismo secundário. As espécies respondem de forma diversificada às condições ambientais que se desenvolvem, produzindo consequentemente, diferentes quantidades de óleos essenciais.

Resultados semelhantes foram verificados por Deschamps et al. (2008) que constaram em diferentes espécies de Mentha x piperita, M. spicata e $M$. arvensis cultivadas em Pinhais-PR, 
Tabela 1. Teores médios de óleo essencial de folhas frescas de Aloysia triphylla em função de diferentes horários e meses de colheita (average contents of essential oil from fresh leaves of Aloysia triphylla harvested on different times and months). Dois Vizinhos, UTFPR, 2011.

\begin{tabular}{|c|c|c|c|c|c|c|}
\hline \multirow{2}{*}{ Coleta (horas) } & \multicolumn{3}{|c|}{2010} & \multicolumn{3}{|c|}{2011} \\
\hline & out & nov & dez & Jan & fev & mar \\
\hline 8 & $0,22 \mathrm{bA}^{*}$ & $0,38 \mathrm{aA}$ & $0,28 \mathrm{bA}$ & $0,36 \mathrm{aA}$ & $0,40 \mathrm{aB}$ & $0,27 \mathrm{bA}$ \\
\hline 11 & $0,41 \mathrm{aA}$ & $0,34 \mathrm{aA}$ & $0,31 \mathrm{aA}$ & $0,20 \mathrm{bB}$ & $0,29 \mathrm{bC}$ & $0,21 \mathrm{bA}$ \\
\hline 14 & $0,26 \mathrm{bcA}$ & $0,30 \mathrm{bA}$ & $0,43 \mathrm{abA}$ & $0,40 \mathrm{abA}$ & $0,50 \mathrm{aAB}$ & $0,22 \mathrm{bcdA}$ \\
\hline 16 & $0,37 \mathrm{bA}$ & $0,26 \mathrm{bA}$ & $0,29 \mathrm{bA}$ & $0,22 \mathrm{cB}$ & $0,54 \mathrm{aAB}$ & $0,21 \mathrm{cA}$ \\
\hline \multirow[t]{3}{*}{18} & $0,35 \mathrm{bA}$ & $0,35 \mathrm{bA}$ & $0,28 \mathrm{bA}$ & $0,24 \mathrm{bAB}$ & $0,58 \mathrm{aA}$ & $0,23 \mathrm{bA}$ \\
\hline & \multicolumn{6}{|c|}{2011} \\
\hline & abr & mai & & & ago & set \\
\hline 8 & $0,20 \mathrm{bA}$ & $0,09 \mathrm{cB}$ & & & $0,27 \mathrm{bA}$ & $0,23 \mathrm{bA}$ \\
\hline 11 & $0,19 \mathrm{bA}$ & $0,16 \mathrm{bA}$ & & & $0,18 \mathrm{bA}$ & $0,25 \mathrm{bA}$ \\
\hline 14 & $0,15 \mathrm{cdA}$ & $0,20 \mathrm{bcdA}$ & & & $0,20 \mathrm{bcdA}$ & $0,37 \mathrm{bA}$ \\
\hline 16 & $0,18 \mathrm{cdA}$ & $0,12 \mathrm{dA}$ & & & $0,33 \mathrm{bA}$ & $0,41 \mathrm{bA}$ \\
\hline 18 & $0,16 \mathrm{bA}$ & $0,23 \mathrm{bA}$ & & & $0,23 \mathrm{bA}$ & $0,38 \mathrm{bA}$ \\
\hline
\end{tabular}

*Médias seguidas das mesmas letras, maiúsculas nas colunas e minúsculas nas linhas, em cada variável, não diferem estatisticamente pelo teste de Tukey, $\mathrm{p}<0,05$ (means followed by same letters, uppercase in columns and lowercase in rows in each variable are not statistically different by Tukey test, $\mathrm{p}<0,05)$.

que todos os genótipos apresentaram rendimento de óleo essencial superior quando a colheita foi realizada no verão (janeiro), comparativamente ao inverno (junho). Segundo os autores, a precipitação no mês de janeiro foi superior aos demais meses de condução do experimento, o que pode ter contribuído para o desenvolvimento vegetativo superior das espécies e consequentemente maior rendimento de óleo essencial.

$\mathrm{Na}$ estação de verão, a tendência é que as temperaturas, nas primeiras horas do dia contribuam para a não volatilização do óleo essencial das folhas, uma vez que este é facilmente volatilizado em altas temperaturas e baixa umidade (Brant et al., 2008). Além das condições ambientais serem favoráveis nessa época para o crescimento no mês de fevereiro, que apresentou os maiores teores de óleo, verificou-se que nesse mês as plantas estavam com folhas jovens. Brant et al. (2008) observaram comportamento semelhante com a espécie na região de Lavras-MG.

Ventrella (2000) constatou que folhas jovens de Lippia alba produzem significativamente mais óleo em relação às folhas mais velhas, onde pode-se inferir uma relação inversa entre idade da folha e produção de óleo essencial, e não somente com quantidade de ma- terial vegetal e estruturas secretoras. Castro (2001), estudando a produção de biomassa, rendimento e composição química dos óleos essenciais de L. alba em diferentes épocas do ano, concluiu que folhas em estádio inicial de desenvolvimento produzem mais óleo quando comparadas com folhas mais velhas.

Por outro lado, nos meses de maio e junho, estação de outono-inverno na região Sul, verificou-se queda de temperaturas $\left(16,9\right.$ e $\left.14,5^{\circ} \mathrm{C}\right)$ e radiação $(596,5$ e 496,8 $\mathrm{kJ} \mathrm{m}^{-2}$ ). É importante considerar que as plantas de cidró iniciam a perda das folhas nos meses de maio e junho, com uma taxa gradativa de perda das folhas de $40 \%$, havendo ainda folhas suficientes para extração de óleo essencial. No mês de julho, com a ocorrência de geadas, a taxa de perda de folhas foi de $100 \%$, onde não há quantidade suficiente de folhas para extração de óleo essencial. Possivelmente, os teores mais baixos de óleo essencial nos meses de maio e junho podem ser explicados pelas condições climáticas adversas e a senescência das folhas que está relacionado com o desenvolvimento fisiológico da planta, que emite folhas em processo continuado, havendo maturação e posterior senescência das folhas mais velhas que acabam por secar ou cair dos ramos nessa época. Isso implica então, necessariamente, num ajuste de época de colheita, que deve ser realizada antes da senescência das folhas mais velhas.

Verificou-se que a produção de óleo essencial tende a diminuir quando a radiação é menor. A radiação é um importante fator que pode interferir diretamente sobre o crescimento e o desenvolvimento da planta, através da fotossíntese, da modulação do fotoperíodo e da qualidade da luz e indiretamente, pelos efeitos na temperatura, sendo fundamental na produção de biomassa e na qualidade dos óleos essenciais (Sangwan et al., 2001). Nos meses de maio e junho, em função das condições climáticas terem sido desfavoráveis ao crescimento, possivelmente ocorreu ativação do mecanismo natural fonte-dreno, o qual degrada os metabólitos secundários e os direciona para a manutenção do metabolismo primário (Taiz \& Zeiger, 2004).

A menor produção de metabólitos secundários sob baixos níveis de radiação no período do inverno são explicados devido ao fato de que as reações biossintéticas são dependentes de suprimentos de esqueletos carbônicos, realizados por processos fotossintéticos e de compostos energéticos que participam da regulação dessas reações, sendo a resposta dessas reações de maneira 
Tabela 2. Dados climatológicos de temperatura, radiação, precipitação e umidade relativa da cidade de Dois Vizinhos-PR, de janeiro 2010 a dezembro 2011 (climatological data of temperature, radiation, precipitation and relative humidity of Dois Vizinhos, Paraná state, from January 2010 to December 2011). Dois Vizinhos, UTFPR, 2011.

\begin{tabular}{lcccc}
\hline Data & $\begin{array}{c}\text { Temperatura } \\
\text { média }\left({ }^{\circ} \mathbf{C}\right)\end{array}$ & $\begin{array}{c}\text { Radiação } \\
(\mathbf{k J} / \mathbf{m})\end{array}$ & $\begin{array}{c}\text { Precipitação } \\
(\mathbf{m m})\end{array}$ & $\begin{array}{c}\text { Umidade } \\
\text { relativa (\%) }\end{array}$ \\
\hline Janeiro/10 & 23,9 & 922,9 & 293,2 & 81,5 \\
Fevereiro/10 & 24,4 & 948,3 & 216,0 & 79,6 \\
Março/10 & 23,1 & 893,4 & 178,0 & 74,4 \\
Abril/10 & 20,0 & 717,8 & 198,6 & 74,8 \\
Maio/10 & 16,1 & 634,1 & 127,4 & 83,1 \\
Junho/10 & 16,4 & 810,4 & 60,2 & 76,1 \\
Julho/10 & 16,1 & 766,9 & 76,6 & 77,8 \\
Agosto/10 & 17,0 & 714,8 & 34,8 & 65,7 \\
Setembro/10 & 19,8 & 687,8 & 28,0 & 62,5 \\
Outubro/10 & 19,6 & 864,4 & 260,6 & 67,0 \\
Novembro/10 & 21,0 & 1070,5 & 272,6 & 69,3 \\
Dezembro/10 & 22,2 & 1145,4 & 267,6 & 81,1 \\
Janeiro/11 & 23,6 & 995,1 & 198,8 & 81,7 \\
Fevereiro/11 & 23,3 & 830,1 & 186,0 & 84,8 \\
Março/11 & 23,3 & 886,1 & 70,8 & 72,1 \\
Abril/11 & 20,6 & 806,8 & 104,8 & 76,5 \\
Maio/11 & 16,9 & 596,5 & 31,0 & 76,6 \\
Junho/11 & 14,5 & 496,8 & 142,2 & 78,5 \\
Julho/11 & 15,9 & 499,8 & 263,0 & 76,6 \\
Agosto/11 & 16,5 & 653,5 & 251,0 & 75,4 \\
Setembro/11 & 19,0 & 1003,2 & 66,2 & 64,6 \\
Outubro/11 & 20,9 & 912,1 & 271,8 & 69,6 \\
Novembro/11 & 21,6 & 1020,5 & 139,0 & 67,8 \\
Dezembro/11 & 23,2 & 1179,3 & 36,8 & 65,5 \\
\hline & & & &
\end{tabular}

positiva para o metabolismo secundário sob altos níveis de radiação (Taiz \& Zeiger, 2004).

Confirmando os resultados obtidos, Brant et al. (2008) também verificaram redução no teor de óleo essencial de cidró nos meses de junho $(0,133 \%)$ e agosto $(0,020 \%)$, devido às condições climáticas adversas de temperatura média baixa $\left(15,7^{\circ} \mathrm{C}\right)$, baixa precipitação $(22,7 \mathrm{~mm})$ e umidade de $60 \%$, resultando em crescimento lento das plantas. Deschamps et al. (2008) constataram que a diminuição do teor de óleo essencial em Mentha campestris por ocasião do inverno estava relacionado à diminuição da temperatura, radiação e umidade.

No horário de colheita realizado às
11:00 horas da manhã, o mês de outubro resultou em maior teor de óleo essencial $(0,41 \%)$ não diferindo estatisticamente dos meses novembro e dezembro, enquanto os demais meses não diferiram estatisticamente. As condições ambientais nesses meses de colheita foram favoráveis ao crescimento e produção de óleo essencial, com temperaturas amenas de 19,$6 ; 21,0$ e $22,2^{\circ} \mathrm{C}$, respectivamente (Tabela 2 ).

Na região onde o estudo foi realizado, no mês de outubro, as plantas estavam em pleno crescimento, após terem passado por um período de paralisação de crescimento vegetativo, em função das baixas temperaturas e formação de geadas. Observou-se que após esse período, as plantas retomam o cresci- mento de forma lenta em agosto e nos meses de setembro e outubro a emissão de brotações e folhas novas é intensa.

Para o horário das 14:00 horas, o mês de fevereiro apresentou o maior teor de óleo essencial $(0,50 \%)$, sem diferir estatisticamente do mês de janeiro e dezembro; os menores teores de óleo essencial nesse horário foram obtidos no mês de março e abril. Nesse horário de colheita para os meses de dezembro, janeiro e fevereiro, o aumento no teor de óleo essencial pode estar relacionado com um aumento na taxa fotossintética, fornecendo maior quantidade de substrato para o metabolismo respiratório e consequentemente um aumento na biossíntese de compostos secundários. A síntese de metabólitos secundários tem um custo energético alto para a planta, pois requer um fluxo constante de precursores a partir do metabolismo primário, ao mesmo tempo enzimas e cofatores ricos em energia como o ATP e NADPH (Gil et al., 2000).

No horário de colheita das 16:00 horas o mês de fevereiro apresentou o maior teor de óleo essencial $(0,54 \%)$. O menor teor de óleo essencial foi obtido no mês de maio $(0,12 \%)$, não diferindo estatisticamente dos meses de abril e junho. Nesse horário de colheita para o mês de fevereiro observou-se a presença de insetos sugadores, principalmente o percevejo vermelho (Corizus hyoscyami) e polinizadores, o que contribuiu para que o metabolismo secundário direcionado à produção de óleos essenciais fosse priorizado.

Leal et al. (2001) informam que plantas sob estresse podem apresentar uma maior densidade de glândulas produtoras de óleo. Além disso, segundo Taiz \& Zeiger (2004) os terpenos voláteis exercem função protetora. Essas substâncias atuam como repelente de herbívoros ovipositores e atraem inimigos naturais, incluindo insetos predadores e parasitas, que matam os insetos herbívoros, reduzindo assim danos adicionais às plantas (Kessler \& Baldwin, 2001).

Resultados semelhantes foram verificados por Brant et al. (2009) com a mesma espécie, em diferentes horários de colheita (8, 12 e 16 h), e os maiores teores de óleo essencial $(0,177$ e 
Tabela 3. Constituintes químicos do óleo essencial (\%) de Aloysia triphylla em função de diferentes épocas de colheita (outubro, novembro e dezembro/2010, janeiro, fevereiro, março, abril, maio, junho, agosto e setembro/2011) (chemical constituents of essential oil (\%) of Aloysia triphylla for different harvest times (October, November and December/2010, January, February, March, April, May, June, August and September/2011). Dois Vizinhos, UTFPR, 2011.

\begin{tabular}{|c|c|c|c|c|c|c|c|}
\hline \multirow{2}{*}{ Compostos } & \multirow{2}{*}{ *IK } & \multicolumn{4}{|c|}{2010} & \multicolumn{2}{|c|}{2011} \\
\hline & & \multicolumn{2}{|l|}{ out } & nov & $\operatorname{dez}$ & \multirow{2}{*}{$\frac{\text { jan }}{0,60 b}$} & \multirow{2}{*}{$\frac{\text { fev }}{0,64 b}$} \\
\hline$\alpha$-pineno & 960 & \multicolumn{2}{|l|}{$0,90 \mathrm{a}$} & - & - & & \\
\hline Sabineno & 980 & - & & - & - & & $1,75 \mathrm{a}$ \\
\hline$\beta$-pineno & 982 & $1,60 \mathrm{a}$ & & $0,71 b$ & $0,74 b$ & - & $1,81 \mathrm{a}$ \\
\hline Mirceno & 994 & - & & - & - & - & $0,44 \mathrm{a}$ \\
\hline Limoneno & 1020 & $13,52 \mathrm{a}$ & & $14,24 \mathrm{a}$ & $14,10 \mathrm{a}$ & $13,82 \mathrm{a}$ & $15,77 \mathrm{a}$ \\
\hline (E)- $\beta$-ocimeno & 1036 & $0,84 \mathrm{c}$ & & $0,62 \mathrm{c}$ & 0,96b & $0,48 \mathrm{c}$ & $2,40 \mathrm{a}$ \\
\hline Linalol & 1078 & $0,90 \mathrm{a}$ & & $0,93 \mathrm{a}$ & $0,87 \mathrm{a}$ & $0,92 \mathrm{a}$ & $0,87 \mathrm{a}$ \\
\hline Cis-óxido limoneno & 1103 & $0,38 \mathrm{a}$ & & $0,33 \mathrm{a}$ & $0,31 \mathrm{a}$ & $0,37 \mathrm{a}$ & $0,29 a$ \\
\hline Trans-oxido limoneno & 1113 & $1,00 \mathrm{a}$ & & $0,81 \mathrm{a}$ & $0,81 \mathrm{a}$ & $1,01 \mathrm{a}$ & $0,74 \mathrm{a}$ \\
\hline Terpinen-4ol & 1149 & $0,68 b$ & & $0,71 b$ & $1,01 \mathrm{a}$ & $0,75 b$ & $1,07 \mathrm{a}$ \\
\hline$\alpha$-terpineol & 1162 & $0,25 \mathrm{c}$ & & - & $0,44 b$ & $0,36 \mathrm{~b}$ & $1,06 \mathrm{a}$ \\
\hline Neral & 1208 & $28,40 \mathrm{~b}$ & & $28,60 b$ & $28,19 b$ & $29,31 b$ & $25,13 b$ \\
\hline Geranial & 1239 & $36,54 a^{* *}$ & & $36,96 a$ & $35,17 \mathrm{a}$ & $37,16 \mathrm{a}$ & $31,11 \mathrm{a}$ \\
\hline Acetato de geranila & 1363 & $2,68 \mathrm{a}$ & & $2,72 \mathrm{a}$ & $2,32 \mathrm{a}$ & $2,35 \mathrm{a}$ & $1,24 b$ \\
\hline Cariofileno & 1405 & $2,42 \mathrm{a}$ & & $2,57 \mathrm{a}$ & $2,56 \mathrm{a}$ & - & $2,27 \mathrm{a}$ \\
\hline Germacreno D & 1464 & - & & - & $1,66 \mathrm{a}$ & - & $1,72 \mathrm{a}$ \\
\hline Ar-curcumeno & 1468 & $1,35 b$ & & $1,42 b$ & - & $1,62 b$ & $1,42 b$ \\
\hline Zingibereno & 1483 & $0,90 b$ & & $0,80 b$ & $0,95 b$ & $0,22 \mathrm{c}$ & $2,33 a$ \\
\hline Nerolidol & 1558 & $4,37 \mathrm{c}$ & & $4,58 \mathrm{c}$ & $5,23 \mathrm{c}$ & $6,58 b$ & $2,68 \mathrm{~d}$ \\
\hline \multirow[t]{3}{*}{ Total identificado } & & 84,73 & & 96,00 & 95,32 & 95,55 & 94,74 \\
\hline & & \multicolumn{4}{|r|}{2011} & & \\
\hline & mar & abr & mai & & jun & ago & set \\
\hline$\alpha$-pineno & - & - & - & & - & $0,10 \mathrm{c}$ & $0,10 \mathrm{c}$ \\
\hline Sabineno & - & - & $0,34 \mathrm{~b}$ & & - & - & - \\
\hline$\beta$-pineno & $0,73 b$ & $0,76 b$ & $0,83 b$ & & $1,38 \mathrm{a}$ & $0,90 b$ & $1,40 \mathrm{a}$ \\
\hline Mirceno & - & - & - & & - & $0,40 \mathrm{a}$ & $0,40 \mathrm{a}$ \\
\hline Limoneno & $13,35 \mathrm{a}$ & $12,45 \mathrm{a}$ & $9,21 b$ & & $11,20 \mathrm{~b}$ & $9,70 b$ & $12,80 \mathrm{a}$ \\
\hline (E)- $\beta$-ocimeno & $0,47 \mathrm{c}$ & $0,50 \mathrm{c}$ & - & & - & $2,10 \mathrm{a}$ & $1,60 b$ \\
\hline Linalol & $1,05 \mathrm{a}$ & $0,76 b$ & $0,67 b$ & & $0,72 b$ & $0,70 b$ & $0,60 \mathrm{~b}$ \\
\hline Cis-óxido limoneno & $0,29 \mathrm{a}$ & $0,30 \mathrm{a}$ & $0,27 \mathrm{a}$ & & $0,33 \mathrm{a}$ & $0,30 \mathrm{a}$ & $0,40 \mathrm{a}$ \\
\hline Trans-oxido limoneno & $0,68 b$ & $0,60 b$ & $0,44 b$ & & $0,53 b$ & $1,30 \mathrm{a}$ & $1,50 \mathrm{a}$ \\
\hline Terpinen-4ol & $0,77 b$ & $0,75 b$ & $0,85 b$ & & $0,61 b$ & $1,50 \mathrm{a}$ & $1,70 \mathrm{a}$ \\
\hline$\alpha$-terpineol & - & $1,40 \mathrm{a}$ & $1,45 \mathrm{a}$ & & $0,53 b$ & $0,40 \mathrm{~b}$ & $0,30 \mathrm{c}$ \\
\hline Neral & $29,04 b$ & $25,50 \mathrm{~b}$ & $20,30 c$ & & $18,02 \mathrm{c}$ & $35,80 \mathrm{a}$ & $35,50 \mathrm{a}$ \\
\hline Geranial & $37,35 \mathrm{a}$ & $34,40 \mathrm{a}$ & $29,39 b$ & & $26,11 b$ & $27,80 \mathrm{~b}$ & $27,10 b$ \\
\hline Acetato de geranila & $2,70 \mathrm{a}$ & $2,20 \mathrm{a}$ & $1,87 \mathrm{~b}$ & & $2,98 \mathrm{a}$ & $2,00 \mathrm{a}$ & $2,10 \mathrm{a}$ \\
\hline Cariofileno & $1,79 b$ & $1,75 b$ & $1,70 b$ & & $0,54 \mathrm{c}$ & $2,80 \mathrm{a}$ & $2,20 \mathrm{a}$ \\
\hline Germacreno D & - & - & - & & - & - & - \\
\hline Ar-curcumeno & $1,09 \mathrm{c}$ & $5,07 \mathrm{a}$ & $6,24 a$ & & $2,36 b$ & $2,70 \mathrm{~b}$ & $2,20 \mathrm{~b}$ \\
\hline Zingibereno & $0,27 \mathrm{c}$ & - & - & & - & $2,12 \mathrm{a}$ & $1,40 \mathrm{~b}$ \\
\hline Nerolidol & $6,14 b$ & $10,70 \mathrm{~b}$ & $14,90 b$ & & $24,34 \mathrm{a}$ & $2,30 \mathrm{~d}$ & $2,20 \mathrm{~d}$ \\
\hline Total identificado & 95,72 & 97,14 & 88,46 & & 89,65 & 92,92 & 93,50 \\
\hline
\end{tabular}

*IK= índice de Kovats (Kovats' index); - = não detectado (not detected); **Médias seguidas das mesmas letras minúsculas nas linhas, em cada variável, não diferem estatisticamente pelo teste de Tukey, $\mathrm{p}<0,05$ (means followed by same lowercase letters in the lines in each variable are not statistically different by Tukey test, $\mathrm{p}<0,05)$. 
0,209\%) foram encontrados nos horários das 8 e 16 h. Em Lippia alba (quimiotipo citral-limoneno) o horário de colheita das $15 \mathrm{~h}$, tanto na estação seca quanto na chuvosa, resultou em maior teor de óleo essencial e de citral (Nagao et al., 2004). Ehlert et al. (2003) obtiveram maiores produções de óleo essencial de folhas frescas de Lippia alba (quimiotipo carvona-limoneno), colhendo-as às 14:00 e 16:00 horas.

No horário das 18:00 horas, o mês de fevereiro apresentou o maior valor $(0,58 \%)$; os demais meses não diferiram estatisticamente. Possivelmente com os dias mais longos a planta tenha priorizado o metabolismo secundário. Angelopoulou et al. (2002) observaram em Cistus monspeliens, durante o dia nos meses de fevereiro, maio e agosto, o rendimento foi maior às $18 \mathrm{~h}$, correspondendo a 0,$005 ; 0,46$ e $0,11 \%$, respectivamente.

Constatou-se também, que os menores teores de óleo essencial foram encontrados no período de inverno, onde as condições ambientais de temperatura e radiação foram desfavoráveis para o crescimento das plantas. Nessa época, com a formação de geadas as plantas ficaram praticamente sem folhas no mês de julho, e retomaram o crescimento vegetativo nos meses de agosto e setembro. Por outro lado, a estação de primavera-verão foi favorável para produção de óleo.

Segundo Telci et al. (2004), o conteúdo de óleo essencial tende a incrementar com o aumento da temperatura de 30 para $35^{\circ} \mathrm{C}$, devido ao aumento no número de glândulas de óleo por unidade de área foliar, porém, podem ocorrer perdas de metabólitos por volatilização. Verificou-se que a sazonalidade e horário de colheita são fatores de importância, visto que a quantidade e a natureza dos constituintes químicos ativos não são constantes durante o ano. Os resultados obtidos no presente trabalho poderão contribuir para definição de práticas de colheitas, gerando suporte para produzir óleo essencial que atenda aos padrões exigidos pelo mercado e formulações de medicamentos fitoterápicos.

A análise cromatográfica dos óleos essenciais extraídos nos diferentes meses de avaliação revelou grande variabi- lidade na composição química. Os constituintes identificados no óleo essencial de cidró foram geranial (26,11-37,35\%), neral $(18,02-29,31 \%)$, limoneno $(9,21$ $15,77 \%)$, nerolidol (2,68-24,34\%), além de E- $\beta$-ocimeno, zingibereno, cariofileno, $\beta$-pineno, sabineno, germacreno $\mathrm{D}$, ar-curcumeno, acetato de geranila, terpinen-4ol, $\alpha$-terpineol, linalool, trans óxido limoneno, $\alpha$-pineno, mirceno e cis oxido limoneno em menores quantidades (Tabela 3). A mistura dos isômeros (geranial e neral) forma o citral, principal constituinte do óleo essencial do cidró (Lorenzi \& Mattos, 2008).

Nas condições em que o estudo foi realizado, constatou-se os maiores teores de geranial $(37,16$ e $37,36 \%)$ e neral (29,31 e 29,04\%), principalmente nos meses de janeiro e março.

A importância comercial do óleo essencial do cidró depende da percentagem desses dois componentes, bem como a baixa percentagem de outros componentes indesejáveis, como o nerol e geraniol, que são formas oxidativas do neral e geranial, e que afetam a qualidade do óleo essencial (Tabatabaie \& Nazari, 2007). Não foram encontrados esses componentes oxidativos no óleo essencial.

Observou-se também que o geranial e o neral, aumentaram seus teores nos meses em que a temperatura, radiação e precipitação foram mais elevadas, e diminuíram nos meses de maio e julho, quando houve decréscimo na temperatura média, radiação e precipitação.

As condições ambientais associadas ao estádio de desenvolvimento da planta influenciaram também na composição do óleo essencial. De acordo com Santos \& Innecco (2003), no decorrer do dia podem ocorrer variações da intensidade luminosa e da temperatura, atuando diretamente em processos primários, como fotossíntese e respiração, e podem influenciar indiretamente a produção de metabólitos secundários, entre eles os constituintes do óleo essencial, cuja síntese depende de produtos do metabolismo primário. A intensidade da luz pode, também, alterar a produção de óleo essencial através de ativação de enzimas fotossensíveis envolvidas na rota do ácido mevalônico, precursor dos terpenos que são constituintes químicos das essências (Souza et al., 2011).

Conclui-se que o horário de colheita é determinado em função da sazonalidade e das condições climáticas do local. $\mathrm{O}$ mês de fevereiro resultou em maior teor de óleo essencial do cidró nos horários de colheita das 16:00 e 18:00 horas. Os constituintes majoritários do óleo essencial foram o geranial $(36,96 \%)$, neral $(29,31 \%)$, nerolidol $(24,34 \%)$ e limoneno $(15,77 \%)$.

\section{REFERÊNCIAS}

ADAMS RP. 2001. Identification of essential oil components by gas chromatography/mass spectroscopy. Illinois: Allured Publishing. $469 \mathrm{p}$.

ANGELOPOULOU D; DEMETZOS C; PERDETZOGLOU D. 2002. Diurnal and seasonal variation of the essential oil labdanes and clerodanes from Cistus monspeliens L. leaves. Biochemical Systematics and Ecology 30: 189-203.

BHERING SB; SANTOS HG; BOGNOLA IA; CÚRCIO GR; MANZATTO CV; CARVALHO JUNIOR W; CHAGAS CS; ÁGLIO MLD; SOUZA JS. 2008. Mapa de solos do Estado do Paraná: legenda atualizada. Rio de Janeiro: EMBRAPA/IAPAR. 74p.

BRANT RS; PINTO JEBP; BERTOLUCCI SKV; ALBUQUERQUE CJB. 2008. Teor do óleo essencial de cidrão (Aloysia triphylla (L'Hérit) Britton Verbenaceae) em função da variação sazonal. Revista Brasileira de Plantas Medicinais 10: 83-88.

BRANT RS; PINTO JEBP; BERTOLUCCI SKV; SILVAA; ALBUQUERQUE CJB. 2009. Teores do óleo essencial de cidrão (Aloysia triphylla (L'Hérit) Britton Verbenaceae) em diferentes horários de colheita e processamentos póscolheita. Ciência Agrotecnologia 33: 2065 2068.

CARVALHO LM; CASALI VWD.1999. Plantas medicinais e aromáticas: relações com luz, estresse e insetos. Viçosa: UFV. 148p.

CASTRO DM. 2001. Efeito de variação sazonal, colheita selecionada e temperaturas de secagem sobre a produção de biomassa, rendimento e composição de óleos essenciais de Lippia alba NEBr. ex Britt. \& Wilson (Verbenaceae). Botucatu: UNESP. 132p. (Tese doutorado).

CZEPAK MP; CRUCIOL CAC. 2003. Produtividade e composição do óleo essencial de capim-limão (Cymbopogon citratus (DC.) Stapf) em diferentes arranjos espaciais. In: SIMPÓSIO BRASILEIRO DE PLANTAS MEDICINAIS: DIAGNÓSTICO E PERSPECTIVAS, 2. Anais... Campinas: Instituto Agronômico. 113p.

DESCHAMPS C; ZANATTA JL; BIZZO HR; OLIVEIRA MC; ROSWAKA LC. 2008. Avaliação sazonal do rendimento de óleo essencial em espécies de menta. Ciência Agrotecnologia 32: 725-730. 
EHLERT PAD; MING LC; MARQUES MOM; FERNANDES DM; FERRI AF; ROCHA WAT; MEIRELES MAA. 2003. Efeito do horário de colheita sobre a carvona e o limoneno do óleo essencial de erva-cidreira brasileira. Horticultura Brasileira 21: 1-5.

GIL A; GHERSA CM; LEICACH S. 2000. Essential oil yield and composition of Tagetes minuta accessions from Argentina. Biochemical Systematics and Ecology 28: 261-274.

KESSLER A; BALDWIN IT. 2001. Defensive function of herbivore induced plant volatile emissions in nature. Science 291: 2141-2144.

LEAL TCAB; FREITAS SP; SILVA JF; CARVALHO AJC. 2001. Avaliação do efeito da variação estacional e horário de colheita sobre o teor foliar de óleo essencial de capim cidreira (Cymbopogon citratus (DC) Stapf). Revista Ceres 48: 445-453.

LORENZI H; MATOS FJA. 2008. Plantas medicinais no Brasil: nativas e exóticas. Nova Odessa: Plantarum. 544 p.

NAGAO EO; INNECCO R; MATTOS SH;
FILHO SM; MARCO CA. 2004. Efeito do horário de colheita sobre o teor e constituintes majoritários de óleo essencial de Lippia alba (Mill) N.E.Br., quimiotipo citral-limoneno. Revista Ciência Agronômica 35: 355-360.

NEGRELLE RRB; ELPO ERS; RÜCHER NGA. 2005. Análise prospectiva do agronegócio gengibre no estado do Paraná. Horticultura Brasileira 23: 1002-1028.

SANGWAN NS; FAROOQI AHA; SFIABIH F; SANGWAN RS. 2001. Regulation of essential oil production in plants. Plant Growth Regulation 34: 3-21.

SANTOS MRA; INNECO R. 2003. Influência de períodos de secagem de folhas de óleo essencial de erva-cidreira (quimiotipo limonenocarvona). Revista Ciência Agronômica 34: 511.

SAS INSTITUTE. 1999. SAS: user's guide statistics: version 8.0 edition. Cary. $956 \mathrm{p}$.

SOUZA MF; MANGANOTTI SA; SOUZA PNS; MEIRA MR; MATOS CC; MARTINS ER. 2011. Influência do horário de coleta, orientação geográfica e dossel na produção de óleo essencial de Cordia verbenáceae. Revista
Biotemas 24: 9-14.

TABATABAIE SJ; NAZARI J. 2007. Influence of nutrient concentrations and $\mathrm{NaCl}$ salinity on the growth, photosynthesis and essential oil content of peppermint and lemon verbena. Turkish Journal of Agriculture and Forestry 31: 245-253.

TAIZ L; ZEIGER E. 2004. Fisiologia Vegetal. Porto Alegre: Artmed. 719p.

TAVEIRA FSN; LIMA WN; ANDRADE EHA; MAIA JGS. 2003. Seasonal essential oil variation of Aniba canelilla. Biochemical Systematics and Ecology 31: 69-75.

TELCI I.; SAHBAZ NI; GUNGOR Y; TUGAY ME. 2004. Agronomical and chemical characterization of spearmint (Mentha spicata L.) originating in Turkey. Economic Botany 58: 721-728.

VENTRELLA MC. 2000. Produção de folhas, óleo essencial e anatomia foliar quantitativa de Lippia alba (Mill.) NEBr. (Verbenaceae) em diferentes niveis de sombreamento e épocas de colheita. Botucatu: UNESP. 95p. (Tese doutorado). 